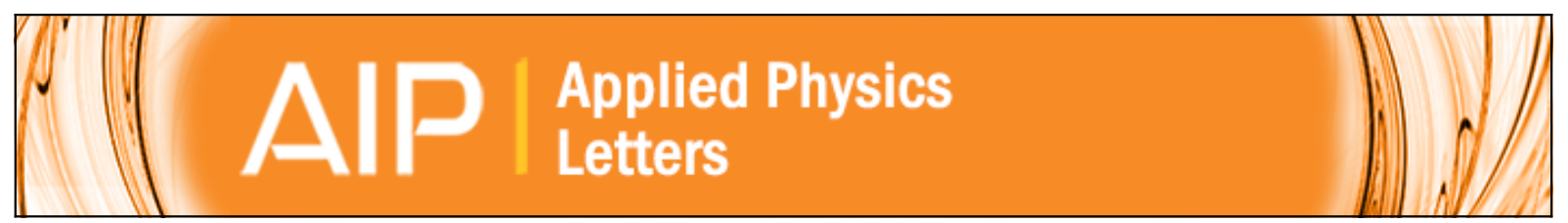

\title{
Positive magnetoresistance in ferromagnetic Nd-doped In2O3 thin films grown by pulse laser deposition
}

G. Z. Xing, J. B. Yi, F. Yan, T. Wu, and S. Li

Citation: Applied Physics Letters 104, 202411 (2014); doi: 10.1063/1.4879463

View online: http://dx.doi.org/10.1063/1.4879463

View Table of Contents: http://scitation.aip.org/content/aip/journal/apl/104/20?ver=pdfcov

Published by the AIP Publishing

\section{Articles you may be interested in}

Defect-band mediated ferromagnetism in Gd-doped $\mathrm{ZnO}$ thin films

J. Appl. Phys. 117, 013913 (2015); 10.1063/1.4905585

Ferroelectric and magnetic properties of Fe-doped $\mathrm{BaTiO} 3$ thin films grown by the pulsed laser deposition J. Appl. Phys. 113, 187219 (2013); 10.1063/1.4801965

Room temperature ferromagnetism in transition metal $(\mathrm{V}, \mathrm{Cr}, \mathrm{Ti})$ doped In 203

J. Appl. Phys. 101, 09N513 (2007); 10.1063/1.2712018

Room temperature ferromagnetism in laser ablated Ni-doped In 203 thin films

Appl. Phys. Lett. 87, 102505 (2005); 10.1063/1.2041822

Fe- and Ni-doped TiO 2 thin films grown on LaAIO 3 and SrTiO 3 substrates by laser ablation Appl. Phys. Lett. 84, 2850 (2004); 10.1063/1.1695103

You don't

still use this

cell phone

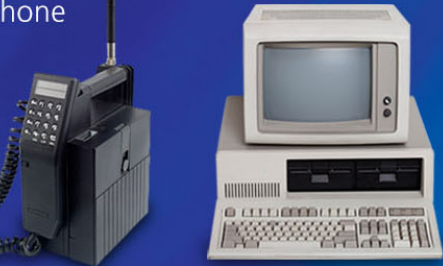

Why are you still using an AFM designed in the 80 's?
It is time to upgrade your AFM

Minimum \$20,000 trade-in discount for purchases before August 31st

Asylum Research is today's technology leader in AFM 


\title{
Positive magnetoresistance in ferromagnetic Nd-doped $\ln _{2} \mathrm{O}_{3}$ thin films grown by pulse laser deposition
}

\author{
G. Z. Xing, ${ }^{1, a)}$ J. B. Yi, ${ }^{1}$ F. Yan, ${ }^{2}$ T. Wu, ${ }^{3}$ and S. $\mathrm{Li}^{1}$ \\ ${ }^{1}$ School of Materials Science and Engineering, The University of New South Wales, Sydney, \\ New South Wales 2052, Australia \\ ${ }^{2}$ School of Engineering and Applied Science, Harvard University, Cambridge, Massachusetts 02138, \\ United States \\ ${ }^{3}$ Division of Physical Sciences and Engineering, King Abdullah University of Science and Technology, \\ Thuwal 23955-6900, Saudi Arabia
}

(Received 8 January 2014; accepted 11 May 2014; published online 23 May 2014)

\begin{abstract}
We report the magnetic and magnetotransport properties of $\left(\operatorname{In}_{0.985} \mathrm{Nd}_{0.015}\right)_{2} \mathrm{O}_{2.89}$ thin films grown by pulse laser deposition. The clear magnetization hysteresis loops with the complementary magnetic domain structure reveal the intrinsic room temperature ferromagnetism in the as-prepared films. The strong $s p-f$ exchange interaction as a result of the rare earth doping is discussed as the origin of the magnetotransport behaviours. A positive magnetoresistance $(\sim 29.2 \%)$ was observed at $5 \mathrm{~K}$ and ascribed to the strong ferromagnetic $s p-f$ exchange interaction in $\left(\operatorname{In}_{0.985} \mathrm{Nd}_{0.015}\right)_{2} \mathrm{O}_{2.89}$ thin films due to a large Zeeman splitting in an external magnetic field of 50 KOe. (C) 2014 AIP Publishing LLC.

[http://dx.doi.org/10.1063/1.4879463]
\end{abstract}

The synergistic combination of electron, charge and spin, which is the root of "spintronics," has the potential to revolutionize electronic devices by effectively utilizing the spin and charge degrees of freedom simultaneously. ${ }^{1}$ Both the fundamental science and the potential applications have been motivating the research efforts focused on materials like dilute magnetic semiconductors $(\mathrm{DMSs})^{2-5}$ and rareearth nitrides, ${ }^{6}$ and so on. Compared with rare-earth nitrides, DMSs have attracted enormous interests because of their potential for innovative magneto-electronic applications. ${ }^{2-5}$ Investigation of potential DMS materials utilizing semiconductor oxide hosts was motivated by both theoretical predictions and experimental findings of ferromagnetism at room temperature in doped oxides. ${ }^{7-11}$ Indium Oxide $\left(\mathrm{In}_{2} \mathrm{O}_{3}\right)$ is one of the most promising oxide hosts for many applications in optoelectronics and transparent electronics. ${ }^{12,13}$ There are several reports of room temperature ferromagnetism in $\mathrm{In}_{2} \mathrm{O}_{3}$-based DMSs, and most of ongoing research efforts focus on the transition metal (TM)-doped $\mathrm{In}_{2} \mathrm{O}_{3},{ }^{14-18}$ where TM ions occupy the corresponding lattice sites in the host with a dilute concentration to minimize the possibility of anti-ferromagnetic ordering. ${ }^{19}$ Compared with the TM counterparts, as the unique dopants, rare earth (RE) elements possess not only great optical properties, ${ }^{20-22}$ but also stronger spin-orbital coupling which warrants further magnetic and magnetotransport studies. ${ }^{7,23}$ However, the reported research in the mechanism of spin-dependent magnetotransport characteristics for the RE-doped $\mathrm{In}_{2} \mathrm{O}_{3}$ is very limited. The understanding of mechanisms underlying the ferromagnetic order may shed light on the ferromagnetism resulted from the hybridization interactions between the weakly localized $s, p$ carriers and the strongly localized $4 f$ electrons. It will answer the key question regarding whether RE doping can produce intrinsic ferromagnetism (FM) with robust magnetotransport properties in wide bandgap oxides, which is

${ }^{a)}$ Electronic mail: guozhong.xing@unsw.edu.au important for developing spintronic materials, in particular for vector control of magnetisation by light. ${ }^{9,24}$

In this work, the structure, magnetic, and magnetotransport characteristics of $\left(\operatorname{In}_{0.985} \mathrm{Nd}_{0.015}\right)_{2} \mathrm{O}_{2.89}$ thin films were investigated. We observed an intrinsic ferromagnetism in the thin films and found a positive magnetoresistance (PMR), which can be explained well by spin-related effect induced by Zeeman splitting. Our complementary experiments reveal that the intrinsic ferromagnetism and the PMR in the thin films stem from the ferromagnetic exchange coupling between the $\mathrm{Nd}$ dopants mediated by the localized carriers and the strong $s p-f$ exchange interaction, respectively.

The target materials were first prepared by sintering the commercial pure $\mathrm{In}_{2} \mathrm{O}_{3}(99.999 \%$, Aldrich) and the mixed powders of $\mathrm{In}_{2} \mathrm{O}_{3}$ and $\mathrm{Nd}_{2} \mathrm{O}_{3}$ (99.999\%, Aldrich). All thin film samples were grown on $\mathrm{Si}$ (100) substrates by a pulsed laser deposition (PLD) system at room temperature followed by in-situ post-annealing treatments at 450,700 , and $900^{\circ} \mathrm{C}$, respectively. The $\mathrm{KrF}$ excimer laser was operating at $248 \mathrm{~nm}^{25}$ and a fluence of $2.66 \mathrm{~J} / \mathrm{cm}^{2}$ with a repetition rate of $5 \mathrm{~Hz}$. The oxygen partial pressure was kept at $2 \times 10^{-1} \mathrm{~Pa}$. The crystallographic structures of thin films were characterized using x-ray diffraction (XRD) with $\mathrm{Cu} \mathrm{K} \alpha$ radiation $(\lambda=0.15418 \mathrm{~nm})$. The morphology, composition, and elements distribution were examined by employing scanning electron microscopy (SEM) and secondary ion mass spectrometry (SIMS) measurements, respectively. The chemical bonding states and the composition of samples were determined by $\mathrm{x}$-ray photoelectron spectroscopy (XPS) using Al $\mathrm{K} \alpha$ source $(\mathrm{h} v=1486.68 \mathrm{eV})$. Magnetic properties were measured by a superconducting quantum interference device (SQUID) magnetometer. The magnetic domain structures were investigated by magnetic force microscope (MFM) scanning on an Asylum setup. Further electrical and magnetotransport properties were performed on the samples with the van der Pauw configuration in a Physical Properties Measurement System (PPMS). Throughout the synthesis and 

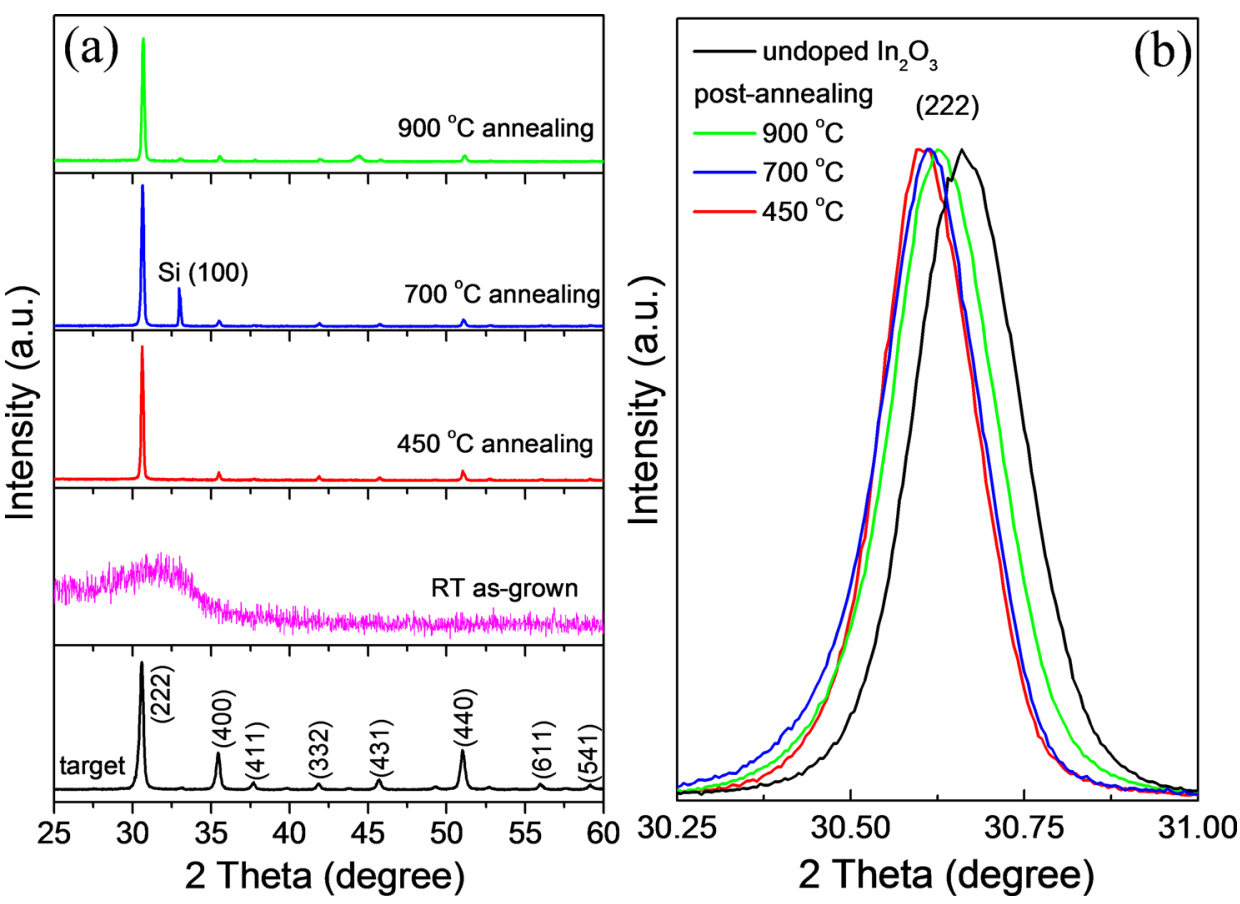

FIG. 1. (a) XRD patterns and (b) enlarged preferred orientation (111) diffraction peaks of as-sintered $\mathrm{Nd}$ doped $\mathrm{In}_{2} \mathrm{O}_{3}$ target, as-grown and post-annealed Nd-doped $\operatorname{In}_{2} \mathrm{O}_{3}$ thin films. measurements, only plastic tweezers were used to avoid any potential contamination from metal tools.

Figure 1(a) shows the XRD patterns of the sintered target, as-grown, and post-annealed thin film samples. Clearly, the as-grown thin film sample at room temperature exhibits an amorphous phase with a broad diffraction peak. After post-annealing treatments at higher temperatures, the films possess the cubic bixbyite $\mathrm{In}_{2} \mathrm{O}_{3}$ structure. ${ }^{26}$ In addition, no secondary phase is detected with XRD characterization. Both the undoped and Nd-doped $\mathrm{In}_{2} \mathrm{O}_{3}$ thin films were single phase with a typical cubic structure. The strong and sharp (222) diffraction peaks indicate that the thin films were well crystallized and grown along with the crystallographic orientation of [111]. As shown in Fig. 1(b), (222) peaks of Nd-doped $\mathrm{In}_{2} \mathrm{O}_{3}$ thin films shift towards lower diffraction angle as compared to undoped $\mathrm{In}_{2} \mathrm{O}_{3}$, demonstrating a lattice constant expansion. The shifting of (222) towards lower diffraction angle is due to the fact that the four-fold coordinated ionic radii of $\mathrm{Nd}^{3+}(0.112 \mathrm{~nm})$ is larger than that of $\mathrm{In}^{3+}$ $(0.094 \mathrm{~nm}) .^{27}$ However, with the increase of the annealing temperature, the deviation of $2 \theta$ shifting of (222) from the undoped samples decreased, suggesting that the higher post-annealing temperature favours a better crystallization with the initial strain/stress being released. In this case, we used the Nd-doped $\mathrm{In}_{2} \mathrm{O}_{3}$ sample, which was post-annealed at $900^{\circ} \mathrm{C}$, to study the doping effects on the structure and magnetotransport behaviours of the material due to its best crystal quality.

Figures 2(a) and 2(b) show the high resolution $\mathrm{O} 1 \mathrm{~s}, \mathrm{Nd}$ $3 \mathrm{~d}_{3 / 2}$, and $\mathrm{Nd} 3 \mathrm{~d}_{5 / 2}$ core-level XPS spectra with the characteristic peaks located at $531.14 \mathrm{eV}, 982.4 \mathrm{eV}$, and $1003.7 \mathrm{eV}$, respectively. ${ }^{28}$ All samples were etched by $\mathrm{Ar}^{+}$ions for 2 min first to eliminate the potential surface contamination. The peak positions were calibrated to the adventitious $\mathrm{C} 1 \mathrm{~s}$ peak at $285.0 \mathrm{eV}$. The peak of $\mathrm{O} 1 \mathrm{~s}$ at the higher energy is attributed to absorbed oxygen [Fig. 2(a)]. The $\mathrm{Nd} 3 \mathrm{~d}_{3 / 2}$ and $\mathrm{Nd} 3 \mathrm{~d}_{5 / 2}$ spectra are different from those of $\mathrm{Nd}_{2} \mathrm{O}_{3}$ and in agreement with the previous reports, ${ }^{29}$ suggesting the substitution of $\mathrm{In}$ by $\mathrm{Nd}^{3+}$. Fitting to the integrated peak areas using the calibrated atomic sensitivity factors yields a nonstoichiometric formula of $\left(\operatorname{In}_{0.985} \mathrm{Nd}_{0.015}\right)_{2} \mathrm{O}_{2.89}$, i.e., a $\mathrm{Nd}$ doping level of 1.5 at. \% accompanies with a certain level of

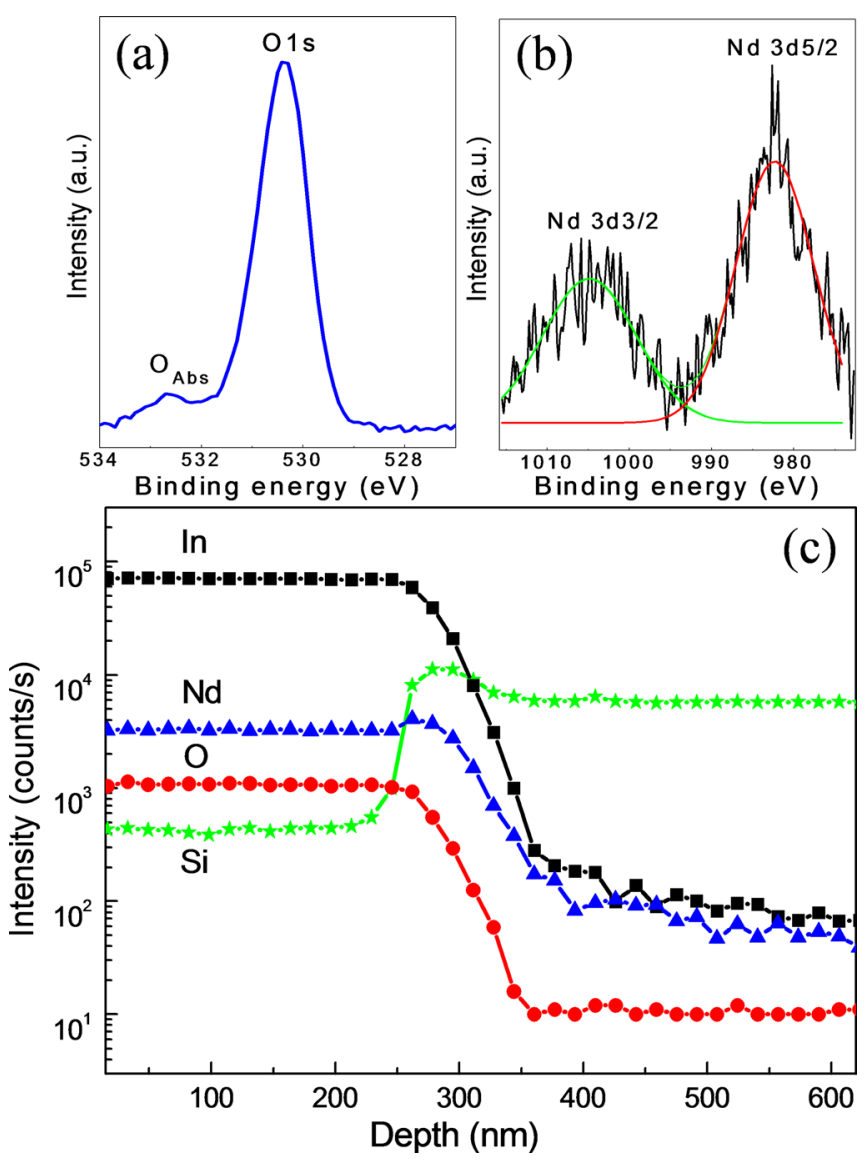

FIG. 2. XPS core-level spectra of (a) $\mathrm{O} 1 s$ and (b) $\mathrm{Nd} 3 d$ for Nd-doped $\mathrm{In}_{2} \mathrm{O}_{3}$ thin film annealed at $900^{\circ} \mathrm{C}$. (c) SIMS depth profiles for all the detected elements in $\left(\operatorname{In}_{0.985} \mathrm{Nd}_{0.015}\right)_{2} \mathrm{O}_{2.89}$ thin film. 
oxygen deficiency. We also carried out the energy-dispersive $\mathrm{x}$-ray spectroscopy composition analysis experiments on Nd-doped $\mathrm{In}_{2} \mathrm{O}_{3}$ sample annealed at $900{ }^{\circ} \mathrm{C}$, and the calculated overall chemical composition match well with the XPS results. The field emission-SEM images of cross-sectional region of $\left(\operatorname{In}_{0.985} \mathrm{Nd}_{0.015}\right)_{2} \mathrm{O}_{2.89}$ thin film indicates a compact and uniform thin film layer deposited on the substrate with a sharp interface and crack/porous free structure (not shown here). Considering that SIMS is a powerful high resolution technique to probe the element distribution, we performed SIMS measurements on our thin film samples using $3 \mathrm{keV}$ $\mathrm{Ar}^{+}$as primary ions. Fig. 1(c) illustrates the SIMS depth profiles of $\mathrm{In}, \mathrm{Nd}$, and $\mathrm{O}$ elements in $\left(\mathrm{In}_{0.985} \mathrm{Nd}_{0.015}\right)_{2} \mathrm{O}_{2.89}$ thin film, suggesting uniform distribution of elements throughout the film. Furthermore, except for In and Nd, there is no other signal of trace amount metallic impurity being probed within the detection limit, which is consistent with the aforementioned XPS results.

Figure 3 shows a comparative study of the magnetic properties in the $\left(\operatorname{In}_{0.985} \mathrm{Nd}_{0.015}\right)_{2} \mathrm{O}_{2.89}$ film measured by the complementary SQUID and MFM techniques. Fig. 3(a) depicts the magnetization versus applied magnetic-field $(M-H)$ with a clear hysteresis loop after subtracting the substrate contribution measured at $300 \mathrm{~K}$, suggesting the room temperature ferromagnetism (RTFM). In contrast, the diamagnetic signal was observed in undoped sample as indicated in the upper inset of Fig. 3(a). In order to exclude the effect of any potential contamination of the substrates that may contribute to the observed magnetism, we measured the bare silicon substrates following the same procedures and their contributions were determined to be solely diamagnetic. For the $\left(\operatorname{In}_{0.985} \mathrm{Nd}_{0.015}\right)_{2} \mathrm{O}_{2.89}$ sample, the coercive field and the saturation magnetization $\left(M_{\mathrm{S}}\right)$ at $300 \mathrm{~K}$ are $135 \mathrm{Oe}$ and $0.1615 \mu_{\mathrm{B}} / \mathrm{FU}$ (i.e., $\mu_{\mathrm{B}} /$ formula unit, the body-centered-cubic bixbyite Indium Oxide structure features with space group $I a \overline{3})$, respectively. This spin magnetic moment is slightly smaller than that of total spin and orbital magnetic moment of free $\mathrm{Nd}^{3+}$ ion due to the hybridization with $\mathrm{O}$ ligand. ${ }^{7,30}$ It is noted that the repeated measurements span over three months confirm the reproducibility of the RTFM result. Furthermore, the temperature-dependent magnetization in the $\left(\operatorname{In}_{0.985} \mathrm{Nd}_{0.015}\right)_{2} \mathrm{O}_{2.89}$ sample was measured under an applied magnetic field of 500 Oe in both the zero-fieldcooled (ZFC) and the field-cooled (FC) modes. The lower inset of Fig. 3(a) suggests that the magnetization starts to increase slightly as $T$ increases from $5 \mathrm{~K}$, and after another sharp drop at $330 \mathrm{~K}$ the magnetization vanishes almost completely at about $375 \mathrm{~K}$, which could be taken as the transition temperature $T_{\mathrm{C}}$ (i.e., Curie temperature).

The observed RTFM in $\left(\operatorname{In}_{0.985} \mathrm{Nd}_{0.015}\right)_{2} \mathrm{O}_{2.89}$ thin film was further confirmed by the room-temperature MFM measurements under the non-contact mode, where the tip-sample magnetic interactions are detected and used to reconstruct the magnetic structure of the sample surface. In MFM measurements, the frequency shift of the tip is proportional to the out-of-plane component of stray field gradient and the MFM tip moment as follows: ${ }^{31}$

$$
\Delta f \propto \partial_{Z} F_{Z}=m_{Z} \partial_{Z}^{2} B_{Z}
$$

where $m_{Z}$ is the magnetic moment of the tip (approximated as a point dipole), $\partial_{Z}^{2} B_{Z}$ is the out-of-plane component of stray field gradient. The topographical and the corresponding MFM images were taken with an image size of $4.5 \times 3.5 \mu \mathrm{m}^{2}$. As shown in Fig. 3(c), there is a clear magnetic response with the distinct phase contrast, indicating RTFM in $\left(\mathrm{In}_{0.985} \mathrm{Nd}_{0.015}\right)_{2} \mathrm{O}_{2.89}$ thin film. This contrast is consistent with the SQUID results and excludes the effects of crosstalk and perturbation from the MFM tip. It is noteworthy that there are some particles with large heights in the topology image as indicated by two open-circle symbols in Fig. 3(b). However, these particles exhibit no magnetic contrast in Fig. 3(c), further revealing the intrinsic nature of the observed RTFM. Such an intrinsic RTFM in $\left(\operatorname{In}_{0.985} \mathrm{Nd}_{0.015}\right)_{2} \mathrm{O}_{2.89}$ is closely associated with its electronic band structure, favouring the mechanism of bound magnetic polarons (BMP), ${ }^{32}$ which are resulted from the spin coupling of intrinsic defects (i.e., oxygen vacancies) with $\mathrm{Nd}$ dopants. Briefly, it is believed that an oxygen vacancy in $\left(\mathrm{In}_{0.985} \mathrm{Nd}_{0.015}\right)_{2} \mathrm{O}_{2.89}$ can trap one electron which is weakly localized. In our $\left(\mathrm{In}_{0.985} \mathrm{Nd}_{0.015}\right)_{2} \mathrm{O}_{2.89}$ thin films, the localized carriers should be the electrons from oxygen vacancies induced by $\mathrm{Nd}$
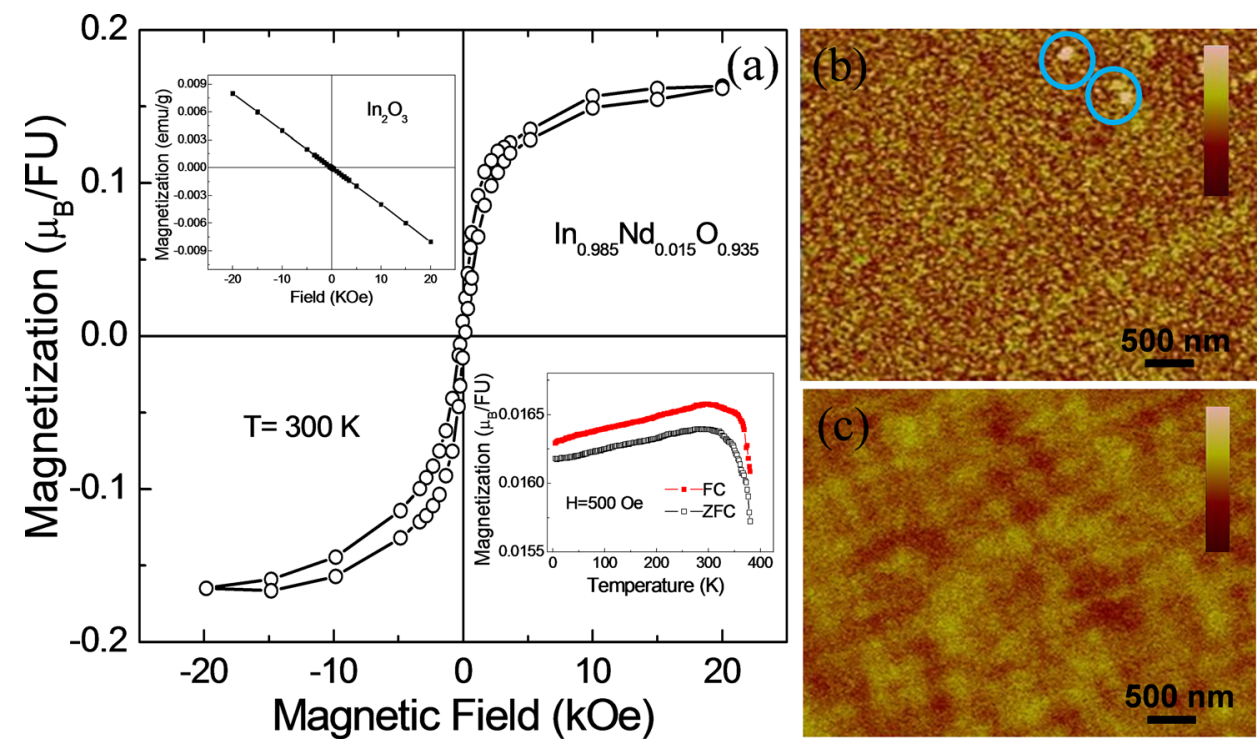

FIG. 3. (a) $M-H$ curves measured at $300 \mathrm{~K}$ on the $\left(\mathrm{In}_{0.985} \mathrm{Nd}_{0.015}\right)_{2} \mathrm{O}_{2.89}$ thin film. Upper inset demonstrates the corresponding $M-H$ loops of undoped $\mathrm{In}_{2} \mathrm{O}_{3}$ thin film. Lower inset shows $M-T$ curves obtained in the temperature range of $5-380 \mathrm{~K}$ under $\mathrm{FC}$ and ZFC measurement modes. (b) Topographic and (c) MFM images of the $\left(\mathrm{In}_{0.985} \mathrm{Nd}_{0.015}\right)_{2} \mathrm{O}_{2.89}$ thin film. 
dopants, which were verified from XPS studies. Such localized carriers will polarize the surrounding magnetic ions of $\mathrm{Nd}^{3+}$ to form the magnetic polarons. ${ }^{32,33}$ When the density of such magnetic polarons reaches a certain threshold, the overlapping of magnetic polarons will induce the spin-spin interaction between $\mathrm{Nd}_{\mathrm{In}}^{3+}$ magnetic ions, and eventually establish the long-range magnetic orderings. The RTFM in $\left(\mathrm{In}_{0.985} \mathrm{Nd}_{0.015}\right)_{2} \mathrm{O}_{2.89}$ might also link with the magnetotransport characteristics of fluctuation-driven BMP which will be discussed in the following. ${ }^{24}$

To verify the scenario of magnetic polarons with localized carriers, we carried out transport measurements on the $\left(\mathrm{In}_{0.985} \mathrm{Nd}_{0.015}\right)_{2} \mathrm{O}_{2.89}$ films. The temperature-dependent resistivity data in Fig. 4(a) suggest a semiconducting behaviour for the sample. The higher resistivity in $\left(\mathrm{In}_{0.985} \mathrm{Nd}_{0.015}\right)_{2} \mathrm{O}_{2.89}$ sample implies a lower density of state at the Fermi level and/or a stronger localization. The electrical transport properties of $\left(\mathrm{In}_{0.985} \mathrm{Nd}_{0.015}\right)_{2} \mathrm{O}_{2.89}$ films were measured by four-probe method by using PPMS. The applied field and sensing current were parallel in the plane of film. Both $\rho v s$. $\mathrm{T}$ curves collected with and without the applied filed of $50 \mathrm{KOe}$ exhibit the semiconductor transport behaviours, showing that the resistance increased as the temperature decreased. We examined various conduction mechanisms and found that our transport data in the low temperature regime reconcile with the typical three-dimensional variable-range-hopping (VRH) mechanism ${ }^{35}$ as shown in the inset of Fig. 4(a), which plots $\ln \rho v$. $\mathrm{T}^{-1 / 2}$. Such transport characteristics of $\left(\mathrm{In}_{0.985} \mathrm{Nd}_{0.015}\right)_{2} \mathrm{O}_{2.89}$ is consistent with the previous reports of Co-doped $\mathrm{ZnO}$ thin films. ${ }^{36-38}$ On the other hand, the thermally activated band conduction appears to be the dominant mechanism in the higher temperature range. ${ }^{39}$

As shown in Fig. 4(b), no room temperature MR was observed because the thermal fluctuations of carriers could be dominant over $s p-f$ interactions at the high temperatures. In contrast, there is a $\sim 29.2 \%$ PMR for $\left(\operatorname{In}_{0.985} \mathrm{Nd}_{0.015}\right)_{2} \mathrm{O}_{2.89}$ thin film measured at $5 \mathrm{~K}$ in an external magnetic field of 50 KOe. It was reported that the PMR is a signature of the fluctuation-driven bound magnetic polaron hopping. ${ }^{34}$ This effect is related to the vector nature of the magnetic order parameter affected by the presence of the localized-electron spin. A PMR is usually attributed to the giant spin splitting of band states caused by $s p-d$ exchange interaction in $3 d$ TM doped DMSs. ${ }^{40}$ In our experiment, for the $\left(\operatorname{In}_{0.985} \mathrm{Nd}_{0.015}\right)_{2}$ $\mathrm{O}_{2.89}$ thin films, $\mathrm{O}$ vacancies and $\mathrm{Nd}$ dopants can supply $s, p$ spin carriers and local spins, respectively. The Zeeman splitting gap of the hopping $s, p$ carriers in a certain fields might be comparable to the thermal energy $\left(k_{B} T\right)$ if the $s, p$ electrons are strongly exchange coupled to the local spins via the ferromagnetic $s p-f$ exchange interaction. In this case, the relatively large Zeeman splitting is believed to be responsible for a PMR, which can be explained as a spin effect caused by the suppression of spin-dependent hopping paths. At the low magnetic field, the PMR can be expressed as ${ }^{38}$

$$
\ln \left(\frac{\rho(H, T)}{\rho(0, T)}\right) \propto \frac{\xi^{4}}{\lambda^{4}}\left(\frac{E_{H}}{k_{B} T}\right)^{3 v}
$$

where $\xi$ is the localization length of carriers, $\lambda=(c \hbar / e H)^{1 / 2}$ is the characteristic magnetic length, and $v$ is the resistance exponent for the linear dependence of $\ln \rho$ on $T^{-D}$ at zero field. When $v=1$ the Eq. (2) can be simplified as

$$
\ln \left(\frac{\rho(H, T)}{\rho(0, T)}\right) \propto \frac{H^{2}}{T^{3}} .
$$

As shown in Fig. 4(c), this relationship is consistent with the quadratic dependence of resistance on the magnetic field in the weak field range, originating from the shrinkage of electron wave functions. ${ }^{35}$ While at the high magnetic field, the carriers will be confined by magnetic field in a much narrower region in the transverse direction than the Coulomb
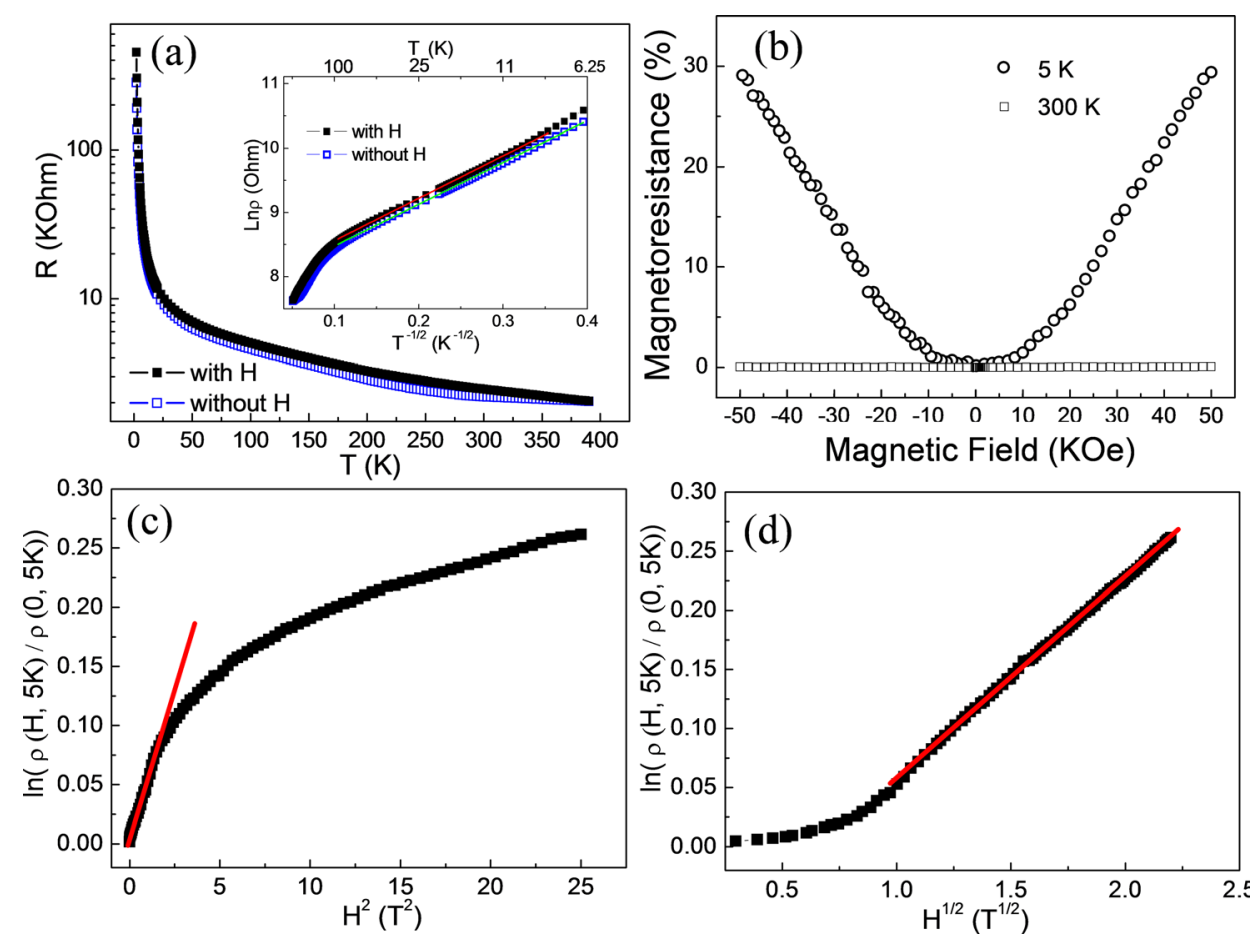

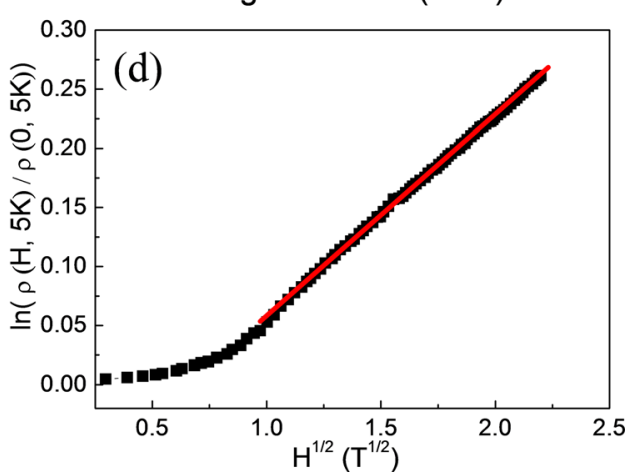

FIG. 4. (a) The resistances as function of temperature between 5 and $380 \mathrm{~K}$ for $\left(\mathrm{In}_{0.985} \mathrm{Nd}_{0.015}\right)_{2} \mathrm{O}_{2.89}$ thin film with and without external magnetic field (50 KOe). Inset shows the plot of $\ln \rho$ vs. $\mathrm{T}^{-1 / 2}$. (b) The MR curves measured at 5 and $300 \mathrm{~K}$, respectively. The field dependence of $\ln (\rho(\mathrm{H}, 5 \mathrm{~K}) /(0,5 \mathrm{~K}))$ as a function of (c) $\mathrm{H}^{2}$ and (d) $\mathrm{H}^{1 / 2}$ for $\left(\mathrm{In}_{0.985} \mathrm{Nd}_{0.015}\right)_{2} \mathrm{O}_{2.89}$ thin film. Solid red lines are guides to the eyes. 
potential imposes. ${ }^{41}$ In this case, Eq. (3) can be expressed as $\ln (\rho(H, T) / \rho(0, T)) \propto f(H) / T^{3}$, similar to a high-field situation with a linear dependence relationship as shown in Fig. 4(d), wherein the behaviour of $f(H) \sim H^{1 / 2}$ clearly reveals the MR characteristics in the high magnetic field.

In summary, the microstructure, magnetic, and transport properties of $\left(\mathrm{In}_{0.985} \mathrm{Nd}_{0.015}\right)_{2} \mathrm{O}_{2.89}$ thin films were studied systematically. The detailed investigations on the structure and chemical composition demonstrate the high quality growth of thin films without cluster and secondary phase formation. The analysis shows that the magnetotransport behaviours of the films were originated from the strong $s p-f$ exchange interaction and a positive magnetoresistance $(\sim 29.2 \%)$ was found to suppress the spin-dependent hopping paths with a large Zeeman splitting at $5 \mathrm{~K}$ in an external magnetic field of $50 \mathrm{KOe}$. This work may open the way towards applying polarized transparent magnetic oxides in future spintronic devices.

This work was supported by the Vice-Chancellor's Research Program (SIR50/PS26746), Faculty of Science Research Grant (IR001/PS30560) and ARC FT100100956 and DP110105338 at University of New South Wales.

${ }^{1}$ S. A. Wolf, D. D. Awschalom, R. A. Buhrman, J. M. Daughton, S. von Molnar, M. L. Roukes, A. Y. Chtchelkanova, and D. M. Treger, Science 294, 1488 (2001).

${ }^{2}$ I. Žutić, J. Fabian, and S. Das Sarma, Rev. Mod. Phys. 76, 323 (2004).

${ }^{3}$ G. Z. Xing, J. B. Yi, J. G. Tao, T. Liu, L. M. Wong, Z. Zhang, G. P. Li, S. J. Wang, J. Ding, T. C. Sum, C. H. A. Huan, and T. Wu, Adv. Mater. 20, 3521 (2008).

${ }^{4}$ T. Dietl, H. Ohno, F. Matsukura, J. Cibert, and D. Errand, Science 287, 1019 (2000).

${ }^{5}$ M. Venkatesan, C. B. Fitzgerald, J. G. Lunney, and J. M. D. Coey, Phys. Rev. Lett. 93, 177206 (2004).

${ }^{6}$ S. Granville, B. Ruck, F. Budde, A. Koo, D. Pringle, F. Kuchler, A. Preston, D. Housden, N. Lund, A. Bittar, G. Williams, and H. Trodahl, Phys. Rev. B 73, 235335 (2006).

${ }^{7}$ D. D. Wang, Q. Chen, G. Z. Xing, J. B. Yi, S. B. Rahman, J. Ding, J. L. Wang, and T. Wu, Nano Lett. 12, 3994 (2012).

${ }^{8}$ M. Venkatesan, C. B. Fitzgerald, and J. M. D. Coey, Nature 430, 630 (2004).

${ }^{9}$ S. B. Ogale, Adv. Mater. 22, 3125 (2010).

${ }^{10}$ J. B. Yi, C. C. Lim, G. Z. Xing, H. M. Fan, L. H. Van, S. L. Huang, K. S. Yang, X. L. Huang, X. B. Qin, B. Y. Wang, T. Wu, L. Wang, H. T. Zhang, X. Y. Gao, T. Liu, A. T. S. Wee, Y. P. Feng, and J. Ding, Phys. Rev. Lett. 104, 137201 (2010).

${ }^{11}$ J. J. Lee, G. Z. Xing, J. B. Yi, T. Chen, S. Li, and M. Ionescu, Appl. Phys. Lett. 104, 012405 (2014).

${ }^{12}$ I. Hamberg and C. G. Granqvist, J. Appl. Phys. 60, R123 (1986).
${ }^{13}$ C. G. Granqvist and A. Hultlker, Thin Solid Films 411, 1 (2002).

${ }^{14}$ N. H. Hong, J. Sakai, N. T. Huong, and V. Brizé, Appl. Phys. Lett. 87, 102505 (2005).

${ }^{15}$ N. H. Hong, J. Sakai, N. T. Huong, A. Ruyter, and V. Brizé, J. Phys.: Condens. Matter 18, 6897 (2006).

${ }^{16}$ G. Peleckis, X. L. Wang, and S. X. Dou, Appl. Phys. Lett. 88, 132507 (2006).

${ }^{17}$ S. J. Hu, S. S. Yan, X. L. Lin, X. X. Yao, Y. X. Chen, G. L. Liu, and L. M. Mei, Appl. Phys. Lett. 91, 262514 (2007).

${ }^{18}$ J. Philip, A. Punnoose, B. I. Kim, K. M. Reddy, S. Layne, J. O. Holmes, B. Satpati, P. R. Leclair, T. S. Santos, and J. S. Moodera, Nature Mater. 5, 298 (2006).

${ }^{19}$ T. Kataoka, Y. Yamazaki, V. R. Singh, A. Fujimori, F.-H. Chang, H.-J. Lin, D. J. Huang, C. T. Chen, G. Z. Xing, J. W. Seo, C. Panagopoulos, and T. Wu, Phys. Rev. B 84, 153203 (2011).

${ }^{20}$ J. Limpert, F. Stutzki, F. Jansen, H.-J. Otto, T. Eidam, C. Jauregui, and A. Tünnermann, Light: Sci. Appl. 1, e8 (2012).

${ }^{21}$ E. M. Dianov, Light: Sci. Appl. 1, e12 (2012).

${ }^{22}$ K. Sugioka and Y. Cheng, Light: Sci. Appl. 3, e149 (2014).

${ }^{23}$ S. Dhar, O. Brandt, M. Ramsteiner, V. F. Sapega, and K. H. Ploog, Phys. Rev. Lett. 94, 037205 (2005).

${ }^{24}$ S. V. Sergeyev, C. B. Mou, E. G. Turitsyna, A. Rozhin, S. K. Turitsyn and K. Blow, Light: Sci. Appl. 3, e131 (2014).

${ }^{25}$ Y.-Y. Lai, Y. P. Lan, and T. C. Lu, Light: Sci. Appl. 2, e76 (2013).

${ }^{26}$ G. Z. Xing, J. B. Yi, D. D. Wang, L. Liao, T. Yu, Z. X. Shen, C. H. A. Huan, T. C. Sum, J. Ding, and T. Wu, Phys. Rev. B 79, 174406 (2009).

${ }^{27}$ R. D. Shannon, Acta Crystallogr. A 32, 751 (1976).

${ }^{28}$ R. K. Selvan, I. Genish, I. Perelshtein, J. M. C. Moreno, and A. Gedanken, J. Phys. Chem. C 112, 1795 (2008).

${ }^{29}$ M. Subramanian, P. Thakur, S. Gautam, K. H. Chae, M. Tanemura, T. Hihara, S. Vijayalakshmi, T. Soga, S. S. Kim, K. Asokan, and R. Jayavel, J. Phys. D: Appl. Phys. 42, 105410 (2009).

${ }^{30}$ J. B. Goodenough, Phys. Rev. 100, 564 (1955).

${ }^{31}$ U. Hartmann, Annu. Rev. Mater. Sci. 29, 53 (1999).

${ }^{32}$ J. M. D. Coey, M. Venkatesan, and C. B. Fitzgerald, Nature Mater. 4, 173 (2005).

${ }^{33}$ D. D. Wang, G. Z. Xing, F. Yan, Y. S. Yan, and S. Li, Appl. Phys. Lett. 104, 022412 (2014).

${ }^{34}$ A. G. Petukhov and M. Foyel, Phys. Rev. B 62, 520 (2000).

${ }^{35}$ G. Z. Xing, Y. H. Lu, Y. F. Tian, J. B. Yi, C. C. Lim, Y. F. Li, G. P. Li, D. D. Wang, B. Yao, J. Ding, Y. P. Feng, and T. Wu, AIP Adv. 1, 022152 (2011).

${ }^{36}$ Y. F. Tian, S.-S. Yan, Q. Gao, J. X. Deng, Y. X. Chen, G. L. Liu, L. M. Mei, and Y. Qiang, Phys. Rev. B 79, 115209 (2009).

${ }^{37}$ Y. F. Tian, W. N. Lin, and T. Wu, Appl. Phys. Lett. 100, 052408 (2012).

${ }^{38}$ M. He, Y. F. Tian, D. Springer, I. A. Putra, G. Z. Xing, E. E. M. Chia, S. A. Cheong, and T. Wu, Appl. Phys. Lett. 99, 222511 (2011).

${ }^{39}$ S. Singh and M. S. R. Rao, Phys. Rev. B 80, 045210 (2009).

${ }^{40}$ M. Sawicki, T. Dietl, J. Kossut, J. Igalson, T. Wojtowicz, and W. Plesiewicz, Phys. Rev. Lett. 56, 508 (1986).

${ }^{41}$ B. I. Shklovskii and A. L. Efros, Electronic Properties of Doped Semi conductors (Springer-Verlag, Berlin, 1984). 